\title{
The random phase approximation applied to ice
}

\author{
M. Macher, ${ }^{1}$ J. Klimeš, ${ }^{1}$ C. Franchini, ${ }^{1}$ and G. Kresse ${ }^{1}$ \\ ${ }^{1}$ University of Vienna, Faculty of Physics and Center for Computational \\ Materials Science, Sensengasse 8/12, A-1090 Vienna, Austria
}

\begin{abstract}
Standard density functionals without van der Waals interactions yield an unsatisfactory description of ice phases, specifically, high density phases occurring under pressure are too unstable compared to the common low density phase $\mathrm{I}_{h}$ observed at ambient conditions. Although the description is improved by using functionals that include van der Waals interactions, the errors in relative volumes remain sizable. Here we assess the random phase approximation (RPA) for the correlation energy and compare our results to experimental data as well as diffusion Monte Carlo data for ice. The RPA yields a very balanced description for all considered phases, approaching the accuracy of diffusion Monte Carlo in relative energies and volumes. This opens a route towards a concise description of molecular water phases on surfaces and in cavities.
\end{abstract}

Copyright (2014) American Institute of Physics. This article may be downloaded for personal use only. Any other use requires prior permission of the author and the American Institute of Physics. The following article appeared in J. Chem. Phys. 140, 084502 (2014) and may be found at http://scitation.aip.org/content/aip/journal/jcp/140/8/10.1063/1.4865748.

\section{INTRODUCTION}

For the last 50 years, water and ice have always been at the forefront of research. With the emergence and establishment of density functional theory (DFT), there has been a slow but continuous shift from empirical force field based methods towards an $a b$ initio description of ice ${ }^{\underline{1}} \underline{\underline{-9}}$ and liquid water ${ }^{10-31}$ However, despite at least three decades of research, an entirely satisfactory parameter free description of energy differences between different water clusters and ice phases has long been unattainable. This changed only recently, with growing compute power making it possible to treat water clusters and solid state phases using $a b$ initio quantum chemical methods ${ }^{32-46}$ and accurate diffusion Monte Carlo (DMC) calculations, $\underline{\underline{7}}$ respectively. These calculations certainly constitute a reference for future work and are important benchmarks for more approximate methods.

What makes the description of ice such a challenge is that the bonding between the water molecules is determined by fairly long range static as well as dynamic (i.e. van der Waals) dipole-dipole interactions and the Pauli exclusion principle between the closed shells at short distances. Van der Waals (vdW) interactions and Pauli repulsion are difficult to handle without explicitly resorting to many electron techniques, such as quantum chemical methods or diffusion Monte Carlo. These methods, in particular the stochastic approaches, are exceedingly demanding when small energy differences between competing phases need to be evaluated with meV accuracy. The quantum chemical methods, on the other hand, are yet restricted to small clusters, requiring fairly complicated incremental approaches for the treatment of three dimensional solids or liquid phases.5,47 Therefore currently DFT methods that are computationally cheap, though more approximate, are widely used. Examples for this are the vdW-DFT of Langreth and Lundqvist and coworkers $48-50$ that employs a non-local density functional and DFT-D using simple pair wise vdW corrections between the constituent $\mathrm{H}$ and $\mathrm{O}$ atoms ${ }^{51-53}$ These approaches are reasonably accurate, and the verdict which one should be preferred over the other is to some extent still debated.

Both vdW-DFT and DFT-D approaches, however, also share the common feature that the exchange interaction, which is an important part of the Pauli exclusion principle, is modeled using DFT. This seems to be problematic, in particular at high densities, where the water molecules approach each other and the molecular charge densities start to overlap. Indeed, both vdWDFT and DFT-D are not entirely satisfactory in capturing the density difference between ambient and high pressure phases $\frac{7}{}$ Also it is not quite obvious whether these two approaches are directly applicable to water on surfaces, a research area receiving currently significant and growing attention; $5-57$ vdW-DFT is fundamentally based on the interaction within jellium, and might work well for ice on metallic surfaces, whereas the addition of pair-wise atom centered vdW potentials is most likely more suitable for water on covalent and possibly ionic substrates.

The random phase approximation (RPA) to the correlation energy avoids these caveats, as it combines the exact exchange with an approximate but reasonably accurate treatment of the correlation. The exact exchange energy is considered to be superior to semi-local exchange functionals at short distances. The correlation part is calculated from the DFT based independent particle response function; for molecules, this yields fairly reliable dispersion forces at large distances with $\mathrm{C}_{6}$ coefficients in good agreement with experiment ${ }^{58-60}$ Current DFT functionals also describe the response of insulators 
and metals reasonably well, and therefore, metals, semiconductors and insulators are handled with about similar accuracy by this approximation. ${ }^{61-64}$ This suggests that the RPA should describe the interaction of water with any substrate reasonably well. What remains to be demonstrated is whether the RPA is accurate for the description of the intermolecular interactions between water molecules. This is exactly the purpose of the present work. Here we apply the Vienna Ab-initio Simulation Package (VASP) to many phases of water ice and compare our findings with well established experimental data and previously calculated DMC data. In general, we find that the RPA results compare very well with the reference data, although the treatment of the repulsive part, the exchange interaction, remains to some extent dependent on the type of orbitals used in the calculations: with DFT orbitals, the exchange interaction is too repulsive yielding too small binding energies and somewhat too large equilibrium lattice constants, whereas with Hartree-Fock orbitals we find opposite trends, too large binding energies and too small lattice constants.

\section{METHODS}

\section{A. Computational Methods}

In the present work, all calculations were performed using VASP. The projector augmented wave method of Blöchl in the implementation of Kresse and Joubert was used $\stackrel{65,66}{ }$ The employed potentials were constructed to conserve the scattering properties of the atoms well up to about 20 Ry above the vacuum level. This was achieved by using additional projectors above the vacuum level. Core radii of 0.95 a.u. for $\mathrm{H}$ and 1.5 a.u. for $\mathrm{O}$ were applied. The scattering properties are, however, correctly described even at much smaller radii of about 1.1 a.u. for $\mathrm{O}$ and 0.5 a.u. for $\mathrm{H}$. Partial waves for $s, p$, and $d$ orbitals were included for both $\mathrm{O}$ and $\mathrm{H}$. Specifically, the O_GW_new and H_GW potentials as released with vasp.5.3 were used.

All plane waves with the kinetic energy lower then $800 \mathrm{eV}$ were used in the DFT calculations. Such a larger cutoff guarantees convergence to a few $\mathrm{meV}$ in absolute energies, and similar results could be obtained at much lower energy cutoffs. However, since DFT calculations are computationally much less expensive than RPA calculations, and in order to avoid tedious convergence tests, we have chosen this rather generous plane wave cutoff. All DFT calculations, except when otherwise noted, are performed using the Perdew, Burke, Ernzerhof functional. $\underline{67}$

Our calculations beyond DFT use two slightly different approximations. The first one is the usual exact exchange (EXX) plus random phase approximation (RPA). In this case, we first perform a standard PBE calculation, and then evaluate the EXX energy using PBE orbitals and add the correlation energy calculated in the random phase approximation with PBE orbitals and PBE oneelectron energies (EXX+RPA@PBE). It has been noted by Ren and coworkers ${ }^{60}$ that this approximation often underestimates the intermolecular binding energies between small molecules, since the occupied PBE orbitals are spatially too delocalized. This results in a too strong Pauli repulsion at the equilibrium distance and, resultantly, too large intermolecular bond lengths. To resolve this issue, various approximations have been suggested among them replacing the exact exchange evaluated employing PBE orbitals by the Hartree-Fock exchange, or a restricted summation of the singles contributions in diagrammatic perturbation theory (rSE) $\underline{60,68}-\underline{\underline{70}}$ We believe that none of these solutions is entirely satisfactory, as all of them assume in essence that the one-particle reduced density matrix $\gamma\left(\mathbf{r}, \mathbf{r}^{\prime}\right)$ from Hartree-Fock is more accurate than the reduced density matrix from the approximate density functional. This might be true for some specific cases, such as small molecules, large band gap insulators, or regions far from any atomic core, however, it can hardly be true for metals or small gap insulators, where present density functionals are far more accurate than the Hartree-Fock approximation. It is, however, clear that the Hartree-Fock orbitals are spatially more contracted than the PBE orbitals, and evaluation of the exact exchange energy with those orbitals hence reduces the intermolecular Pauli repulsion. Consequently the intermolecular distances become smaller, and past experience suggests that this often improves agreement with experiment. $\underline{60}$ To obtain a- what we believe - lower bound for the lattice constants, we have therefore also evaluated the exact exchange energy using Hartree-Fock orbitals. Even in this case, the correlation energy is calculated with PBE orbitals and PBE one electron energies. In this work, we refer to this scheme as HF+RPA@PBE.

The EXX+RPA@PBE and the HF+RPA@PBE calculations are performed at a more modest computational setup than the PBE calculations discussed above. The plane wave energy cutoff for the orbitals was set to $E_{\text {cut }}^{\mathrm{PW}}=450 \mathrm{eV}$. When summations over unoccupied Kohn-Sham states are required (virtual orbitals), all orbitals spanned by the basis set are determined by exact diagonalization of the Kohn-Sham Hamiltonian. The correlation energy in the random phase approximation is then calculated in the usual manner as

$$
E^{\mathrm{RPA}}=\int_{0}^{\infty} \frac{d \omega}{2 \pi} \operatorname{Tr}\left\{\ln \left[1-\chi^{\mathrm{KS}}(i \omega) \nu\right]+\chi^{\mathrm{KS}}(i \omega) \nu\right\}(1)
$$

where $\chi^{\mathrm{KS}}$ is the independent particle response function evaluated using PBE orbitals and one electron energies, and $\nu$ is the Coulomb kernel. The response function itself is also expanded in a plane wave basis set. The plane wave cutoff for this basis set is set to $210-300 \mathrm{eV}$ (smaller than the basis set for the orbitals), and the correlation energy is extrapolated to the infinite basis set limit, assuming that the basis set error falls off like the inverse of the number of plane waves included in the basis set 
for the response function $\underline{58}$ In the VASP code, this requires a single calculation and the extrapolation is performed automatically by the code, requiring a minimum of extra compute time. The structures used for the RPA calculations were determined by completely relaxing all internal parameters of the structures (including the cell shape) at a set of volumes employing the PBE functional (and the previously mentioned cutoff of $800 \mathrm{eV}$ ). In the subsequent RPA calculations, the PBE structures were kept fixed since forces and the stress-tensor are presently not available within the RPA. Similar strategies are also routinely adopted in DMC simulations and most coupled cluster quantum chemistry calculations.

For ice, the RPA energy volume curves usually span a very small energy range of the order of $10 \mathrm{meV}$ per molecule, when the volume varies by $10 \%$. These small energy changes make converged calculations particularly challenging. For instance, when the volume changes, the number of plane waves $\mathbf{G}$ at a $\mathbf{q}$ point in the Brillouin zone changes disruptively with volume:

$$
\frac{\hbar^{2}|\mathbf{G}+\mathbf{q}|^{2}}{2 m_{e}}<E_{\text {cut }} .
$$

This problem is more severe for high symmetry structures than for low symmetry structures, since reciprocal lattice shells show more degeneracies in high symmetry structures. The smoothness can be improved by either increasing the cutoffs (which we found unpractical for the present calculations) or increasing the number of sampling points $\mathbf{q}$ in the Brillouin zone. In this work, we have followed the second approach, i.e. increasing the number of $\mathbf{q}$ points until a smooth energy-volume curve was obtained. For some phases, a sizable residual noise, however, remains, and the bulk moduli might exhibit error bars of about 10-20\% (estimated from different $\mathbf{q}$ point sets).

To obtain the ice binding energies with respect to the free water molecule, accurate reference values for the exchange and correlation energy of the water molecule are required. The exchange energies, EXX and HF, were determined by calculating the energy of a single $\mathrm{H}_{2} \mathrm{O}$ molecule in a box with the box size systematically varied between 7 and $21 \AA$. The correlation energy was determined in a smaller $7 \AA$ box using $3 \times 3 \times 3$ k-points. Although absolute energies are not directly transferable between different codes or potentials we also report the final molecular results obtained by VASP. The final RPA correlation energy of a single water molecule is $-12.426 \mathrm{eV}$, the EXX energy using PBE orbitals and the HF energy are $-29.254 \mathrm{eV}$ and $-29.479 \mathrm{eV}$, respectively. As a matter of fact, the (self-consistently evaluated) HF energy is significantly lower than the EXX energy.

\section{B. Considered Phases}

From the known crystalline phases of ice we have considered the ones listed in Table \ and depicted in fig-

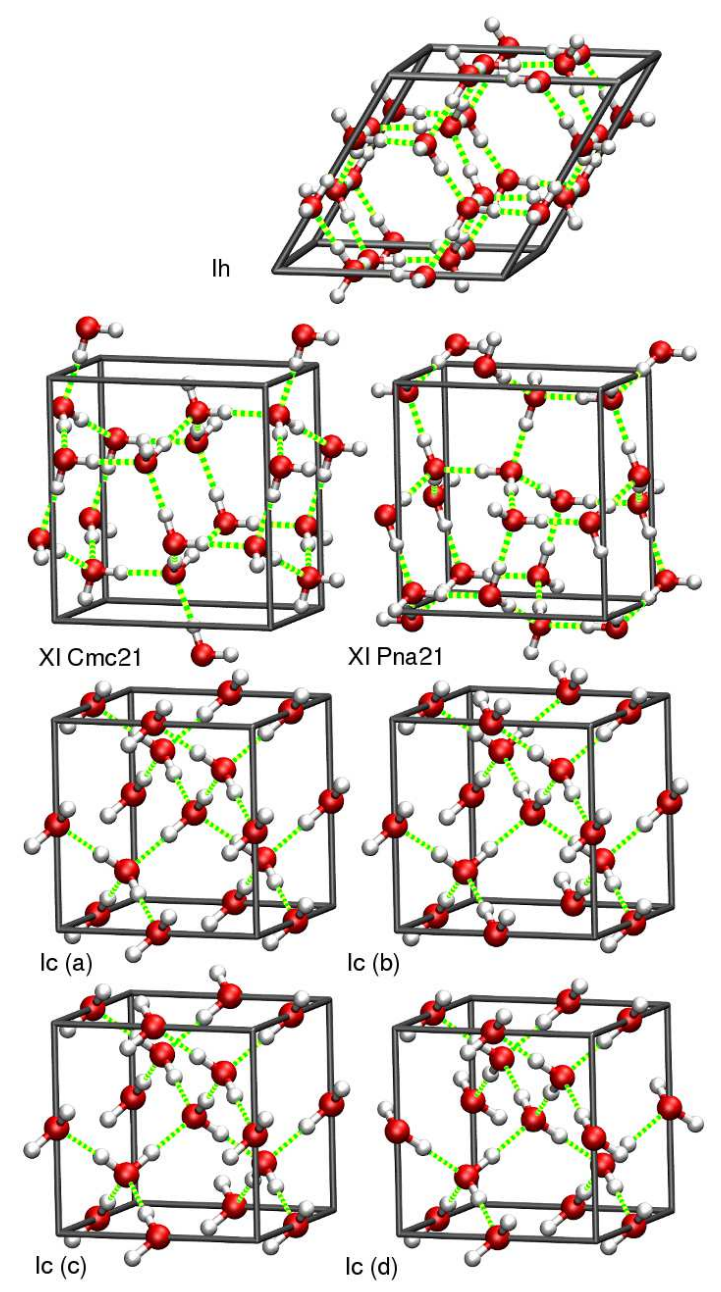

FIG. 1. Low density structures of ice considered in the present work. Large red and small white spheres indicate the oxygen and hydrogen atoms, respectively. Dashed (green) lines indicate hydrogen bonds. Full thick lines demarcate the unit cell.

ures 1 and 2 All structures follow the usual building rules for ice: each water molecule has four nearest neighbor water molecules, donates two hydrogen bonds, and accepts two hydrogen bonds. The hydrogen atoms (protons) are typically not fixed in their high-symmetry positions. This results in the formation of so-called proton disordered phases, i.e. possible configurations of proton positions consistent with the ice rules. The phases of ice under scrutiny in the present work can be arranged into two groups depending on their densities. (i) Lowdensity phases (see Fig. 11): proton disordered hexagonal ice $\left(\mathrm{I}_{h}\right)$, the most stable proton ordered form of $\mathrm{I}_{h}$ (XI $\left.\mathrm{Cmc} 2_{1}\right)$, the second most stable proton ordered phase of $\mathrm{I}_{h}$ (XI Pna2 $2_{1}$ ), and four proton ordered cubic phases $\left(\mathrm{I}_{c}\right.$ a-d). (ii) High-density phases: the proton ordered form of ice III (ice IX), the proton ordered ice II, the proton ordered form of ice V (ice XIII), the proton ordered form of ice VI (ice XV), and the proton ordered form of ice VII 
TABLE I. Summary of ice phases considered in the present work. The experimental Bravais lattice (exp), as well as the considered approximation to the experimental structure (calc) are specified. The total number of molecules is given in the column "mol". The column "k-points" indicates the number of divisions $(n)$ in each reciprocal lattice direction used to generate a uniform k-point grid (e.g. $n \times n \times n)$.

\begin{tabular}{|c|c|c|c|c|c|}
\hline phase & space group & $\exp$ & calc & mol. & k-points \\
\hline XI Cmc $2_{1}$ & $\mathrm{Cmc} 2_{1}(\# 36)$ & orthorhombic & base c. orthorhombic & 4 & 6 \\
\hline $\mathrm{I}_{c}(\mathrm{a})$ & $\mathrm{I} 4_{1}$ md $(\# 109)$ & tetragonal & body c. tetragonal & 2 & 7 \\
\hline $\mathrm{I}_{c}(\mathrm{~b})$ & $\mathrm{Pna}_{1}(\# 33)$ & orthorhombic & simple orthorhombic & 4 & 5 \\
\hline IX & $\mathrm{P} 4_{1} 2_{1} 2(\# 92)$ & tetragonal & simple tetragonal & 12 & 3 \\
\hline II & $\mathrm{R} \overline{3}(\# 148)$ & rhombohedral & trigonal (rhombohedral) & 12 & 3 \\
\hline XIII & $\mathrm{P} 2_{1} / \mathrm{a}(\# 14)$ & monoclinic & simple monoclinic & 28 & 1 \\
\hline XV & P1 (\#1) & triclinic & triclinic & 10 & 3 \\
\hline VIII & I4 1 /amd (\#141) & tetragonal & body c. tetragonal & 4 & 6 \\
\hline
\end{tabular}

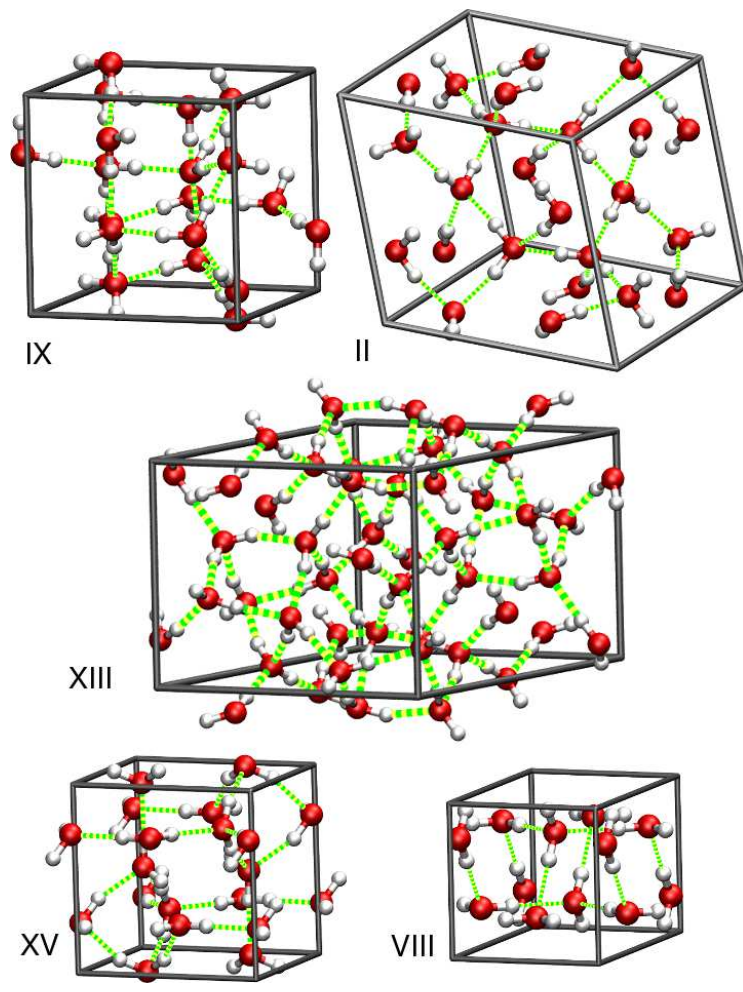

FIG. 2. High density structures of ice considered in the present work. Color coding is the same as in figure 1

(ice VIII). In the following, we will briefly summarize the structural details of each phase.

Hexagonal ice $\left(I_{h}\right)$ is the common phase of ice naturally encountered. The protons are usually disordered, although partial order can be induced by careful, slow annealing in the presence of some ionic "catalyst" such as $\mathrm{KOH}, \underset{71,72}{ }$ which increases the usually slow reorienta- tion rate in ice. To model the disorder, we have adopted the scheme used by Hamann $\stackrel{73}{\underline{3}}$ This lowers the symmetry to monoclinic with a structural model containing 12 molecules. All our energies are referenced to this structure in the final figures. This needs to be considered when comparison is made with other calculations. However, a recent study of Santra et al. suggests that the energy difference to a larger 96 molecule cell is only in the range of $1 \mathrm{meV} \stackrel{74}{\underline{7}}$ To study proton ordered variants of hexagonal ice, we have included one hexagonal structure with ferroelectric and one with anti-ferroelectric order, respectively. The ferroelectrically ordered phase is realized in ice XI with space group $\mathrm{Cmc2} 2_{1}$. The structure was first resolved experimentally by Leadbetter et al $\underline{n 1}^{71}$ showing "polar" order on a length scale of about $40 \AA$. An anti-ferroelectrically ordered phase can be realized in the space group Pna2 $2_{1}$ and was suggested by Davidson et al ${ }^{75}$ Further prototypically ordered structures have been studied systematically in Refs. 76 and 77 using density functional theory and a plane wave code.

Cubic ice $\left(I_{c}\right)$ is experimentally difficult to prepare and forms only under certain conditions, for instance, it is believed to form in the Earth's upper troposphere at temperatures of less then $220 \mathrm{~K} \stackrel{78}{ }$ Above $240 \mathrm{~K}$, cubic ice tends to transform to hexagonal ice. As for hexagonal ice, cubic ice is usually proton disordered. Here we considered four different proton ordered phases out of the 11 different proton configurations enumerated in Ref. 79. The first phase is fully ferroelectrically ordered and denoted as $\mathrm{I}_{c}(\mathrm{a})$, whereas the other three are ferrielectrically (bc) and anti-ferroelectrically (d) ordered; the number of anti-ferroelectrically ordered neighbors increases as one goes from structure (b) to structure (d). Details of the structures are discussed elsewhere .80

Ice IX is stable at temperatures below $140 \mathrm{~K}$ and pressures between 300 and $400 \mathrm{MPa}$. Experimentally it is 
formed by slow cooling of tetragonal crystalline ice III (formed by cooling water down to $250 \mathrm{~K}$ at $300 \mathrm{MPa}$ ) $\underline{81}$ Both disordered ice III and ordered ice IX have the space group $\mathrm{P} 4_{1} 2_{1} 2$ (\#92). At similar pressures (300 MPa) and low temperature $(198 \mathrm{~K})$, ice II (the rhombohedral crystalline form of ice) can be formed from ice $\mathrm{I}_{h}{ }^{82}$ Ice II is a proton ordered phase with space group R $\overline{3}$ (\#148), with no direct proton disordered counterpart $\underline{83}$ The structure is characterized by two hexagonal rings, connected by hydrogen bonds.

The monoclinic proton-ordered ice XIII was successfully prepared and structurally determined by neutron powder diffraction in $2006 . \stackrel{84}{*}$ After doping with $\mathrm{HCl}$, it can be formed from the corresponding proton-disordered monoclinic phase of ice (ice V) at temperatures slightly below $130 \mathrm{~K}$ and applying a pressure of $0.5 \mathrm{GPa}$. In the pressure range from $0.8 \mathrm{GPa}$ to $1.5 \mathrm{GPa}$ and at similar low temperatures, the proton-ordered counterpart to the proton-disordered phase VI has been identified in $2009^{85}$ and named ice XV (triclinic).

The final highest pressure phase considered here is ice VIII. It is the proton ordered form of the proton disordered ice VII with tetragonal space group $\mathrm{I}_{1} /$ amd (\#141). The oxygen sublayer is the same for both structures and all molecules have an equivalent environment. The structure consists of two inter-penetrating, but not interconnected cubic ice $\mathrm{I}_{c}$ sublattices $\stackrel{86}{\underline{86}}$ The sublattices have opposite dipole moments resulting in an antiferroelectric ordering $\underline{87}$

\section{RESULTS}

In the first subsection we present results for few selected phases, elaborating on illustrative tests and important issues. In the subsequent subsections, binding energies and equilibrium volumes are discussed.

\section{A. Preliminary Remarks}

\section{Optimized volume}

To illustrate the general behavior and, specifically, the rather slow k-point convergence, we show in Figs. 3 and 4 the energy as a function of the cell volume per water molecule for different k-point meshes. The data computed within PBE, EXX+RPA@PBE and $\mathrm{HF}+\mathrm{RPA} @ \mathrm{PBE}$ are shown for ice $\mathrm{I}_{h}$ (low density case) in Fig. 3 and for ice VIII (high density case) in Fig. 4.

The first thing to notice is that, for ice $\mathrm{I}_{h}$ at the PBE level, the equilibrium volume is much too small compared to experiment. Explicitly including many-body correlation effects in diagrammatic perturbation theory clearly improves upon this point, with the equilibrium volumes now approaching the experimental values. For the high density ice VIII phase, the different available experimental values for the volume $\left(18.61 \AA^{3} \text {, and } 20.09 \AA^{3}\right)^{88,89}$

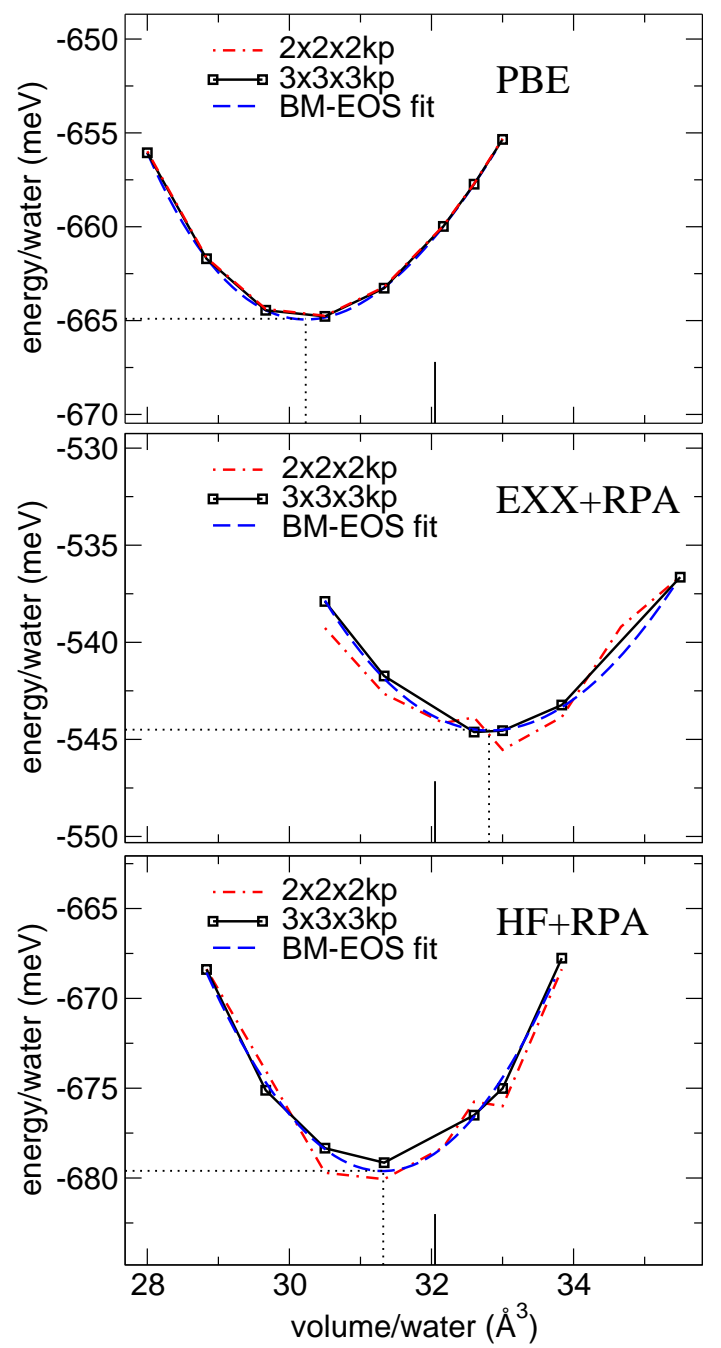

FIG. 3. Binding energy per water molecule versus volume for ice $\mathrm{I}_{h}\left(\mathrm{P}_{3} / \mathrm{mmc}\right)$ for PBE, EXX+RPA@PBE, and $\mathrm{HF}+\mathrm{RPA} @ \mathrm{PBE}$. The experimental volume is shown as a vertical line. The dotted lines indicate the theoretical volume and energy.

hamper a definite assessment of the various theoretical methods. In this case, PBE certainly yields much too large volumes. This can be attributed to the lack of $\mathrm{vdW}$ interactions that become more important at small volumes, where the number of neighbors increases $\underline{\underline{z}}$ Also in this case, the RPA delivers an improved description compared to PBE. The EXX+RPA optimized volume is bracketed by the experimental values, whereas the $\mathrm{HF}+\mathrm{RPA}$ estimate is much closer to the lower experimental value. Following the expectations already outlined in the introduction, the HF+RPA@PBE equilibrium volumes are smaller than the EXX+RPA@PBE volumes. In fact, both, HF+RPA@PBE and EXX+RPA@PBE roughly bracket the experimental volume, something we will also observe for other ice phases. 


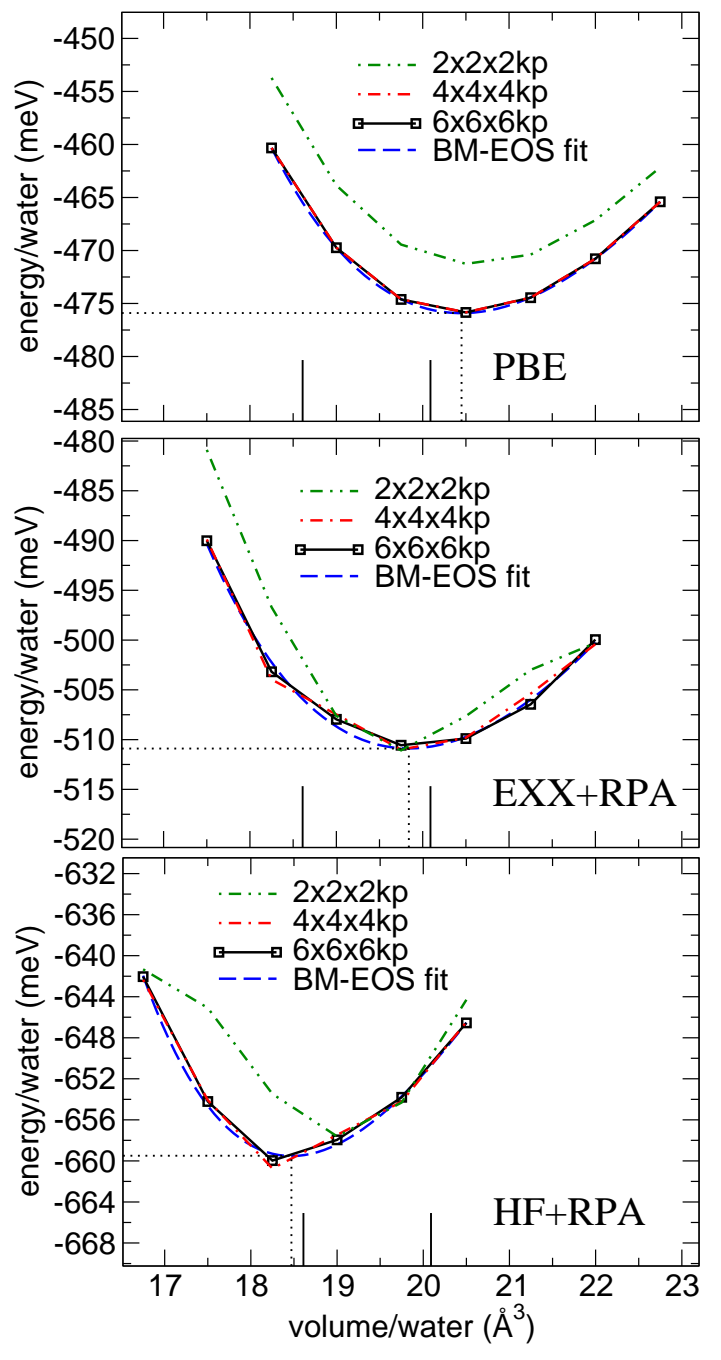

FIG. 4. Binding energy per water molecule versus volume for ice VIII for PBE, EXX+RPA@PBE, and HF+RPA@PBE. The experimental volume is shown as a vertical line.

\section{Convergence with $k$-points}

As for the k-point convergence, we find that the DFT results converge rapidly with $\mathrm{k}$-points, and show only very little jaggedness, whereas the RPA results exhibit quite some residual noise for a coarse $2 \times 2 \times 2 \mathrm{k}$-point grid, especially for the high density phase (ice VIII). The jaggedness is mostly gone in the low density $\mathrm{I}_{h}$ phase with $3 \times 3 \times 3$ points, whereas in ice VIII reasonable smoothness is only achieved from $4 \times 4 \times 4 \mathrm{k}$-points on. For the converged k-point grids, the data can be well fitted with a 3rd order Birch-Murnaghan equation of state.

The final k-point grids for each phase are summarized in Table [ As already mentioned, in some cases a residual jaggedness prevails, since we were unable to increase the k-point grids significantly beyond the values in the Tables with the present code. In any considered case, however, the minimum was clearly resolved in the energy volume curve.

\section{Structure of ice IX}

Ice IX has a tetragonal structure, and initially we performed a structural optimization for all internal parameters (including the $c / a$ ratio) at each volume using the PBE functional. This lead to results in disagreement with the previous calculations. ${ }^{7}$ However, the previously reported calculations were performed with the cell shape fixed to the experimentally determined structure. Relaxation of the lattice shape changes the results only little for all, but the ice IX structure. In fact, PBE yields only a mediocre description of the $c / a$ ratio for ice IX, with the value approaching 1.123 whereas the experimental values are close to $1.01,90,91$ In this case, vdW interactions are particularly important along the $c$ direction, and only inclusion of them improves the $c / a$ ratio. For instance DFT-D yields a $c / a$ ratio around 1.03 . To maintain compatibility with the rest of the results, we decided to stick to the optimization of the structures using the PBE functional, but kept the $c / a$ ratio of ice IX fixed to the experimental value. Both results, the one with the full optimization of the $c / a$ ratio and the one with the $c / a$ ratio fixed to the experimental values are shown in the tables, whereas the figures report on the results for fixed $c / a$ ratio. As Tab. IIIshows, the RPA yields a lower energy with the $c / a$ ratio fixed to the experimental value.

\section{B. Binding energies}

TABLE II. Binding energies in eV per water molecule as obtained for different phases of ice with PBE, EXX+RPA@PBE, and HF+RPA@PBE. The energies were evaluated at the equilibrium volumes corresponding to a given method and phase.

\begin{tabular}{llll}
\hline \hline Phase & $\mathrm{PBE}$ & $\mathrm{EXX}+\mathrm{RPA}^{\mathrm{EXX}}$ & $\mathrm{HF}+\mathrm{RPA}^{\mathrm{HF}}$ \\
\hline $\mathrm{I}_{h}$ & -0.6649 & -0.5445 & -0.6796 \\
$\mathrm{XI} \mathrm{Cmc2}_{1}$ & -0.6678 & -0.5476 & -0.6831 \\
XI Pna2 $_{1}$ & -0.6637 & -0.5443 & -0.6779 \\
$\mathrm{I}_{c}(\mathrm{a})$ & -0.6682 & -0.5478 & -0.6824 \\
$\mathrm{I}_{c}(\mathrm{~b})$ & -0.6654 & -0.5448 & -0.6793 \\
$\mathrm{I}_{c}(\mathrm{c})$ & -0.6641 & -0.5417 & -0.6769 \\
$\mathrm{I}_{c}(\mathrm{~d})$ & -0.6627 & -0.5425 & -0.6773 \\
IX & -0.6199 & -0.5290 & -0.6652 \\
$\mathrm{IX} \mathrm{c/a} \mathrm{exp}$ & -0.6126 & -0.5362 & -0.6735 \\
$\mathrm{II}$ & -0.5918 & -0.5358 & -0.6712 \\
XV & -0.5474 & -0.5223 & -0.6620 \\
VIII & -0.4759 & -0.5111 & -0.6593 \\
\hline \hline
\end{tabular}

The binding energies of all considered phases are summarized in Tab. II and Fig. 5, In the figure, the energies are shown with respect to ice $I_{h}$. Let us first concentrate on the phases at ambient pressure, hexagonal ice and cubic ice (lower panel in Fig. (5). From the figure, it is clear that there is very little difference between the standard 


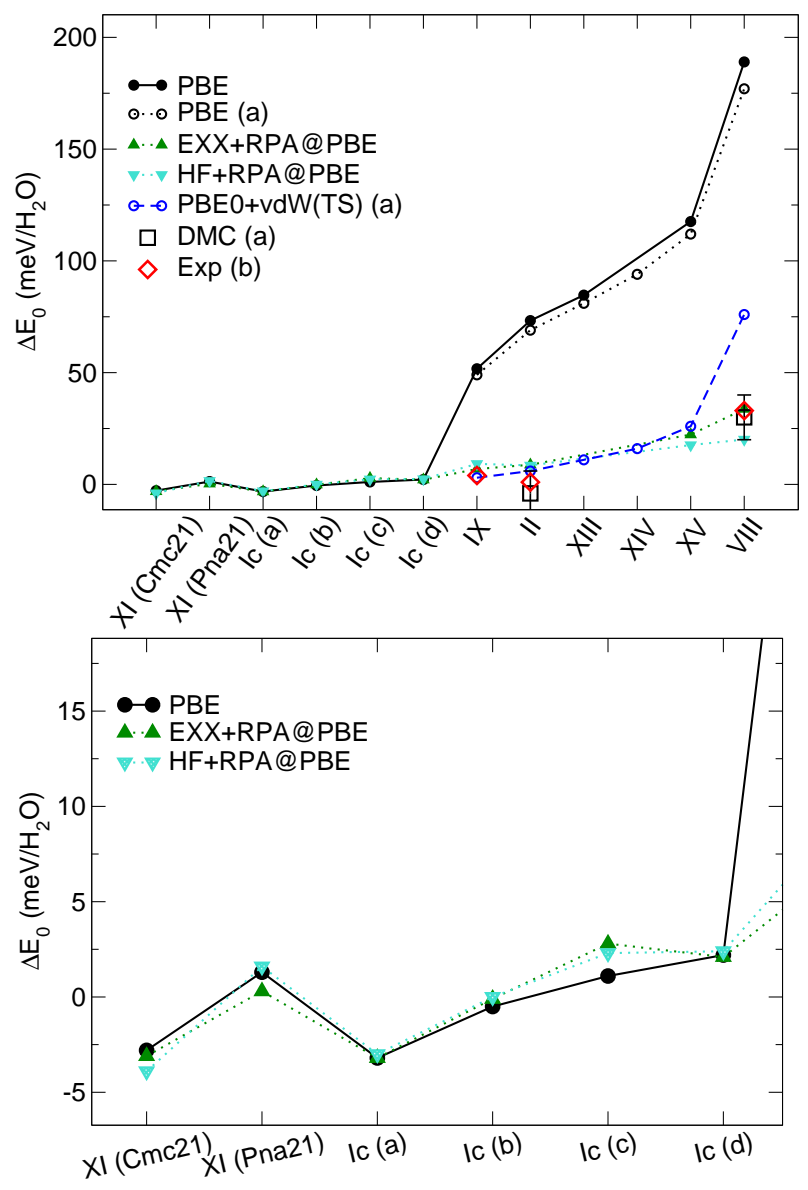

FIG. 5. Binding energies per water molecule relative to ice $\mathrm{I}_{h}$. $\Delta E_{0}$ is the energy with respect to proton disordered $\mathrm{I}_{h}$ for the same method: $\Delta E_{0}=E_{0}$ (phase) $-E_{0}\left(\mathrm{I}_{h}\right)$. Other theoretical data marked with (a) are from Ref. 74 [PBE, PBE0-vdW(TS), DMC]. Experimental data (b) are from Ref. 92 .

density functional theory calculations (PBE) and the two RPA variants for relative energies. Note that, mostly because of the insufficient sampling of the Brillouin zone, the errors in the RPA energies are about $1 \mathrm{meV}$ for relative energies. Clearly the proton disordered hexagonal phase is about $3 \mathrm{meV}$ higher in energy than the ferroelectrically ordered phase XI Cmc $2_{1}$. We note that this is in agreement with the experimental data that also predict a long range ferroelectrically ordered phase as the actual low temperature ground state structure of hexag-

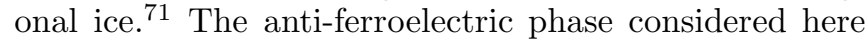
(XI Pna2 $2_{1}$ ) is slightly higher in energy than the disordered phase of Hamann, but the energy difference is as small as $2 \mathrm{meV}$ in PBE and HF+RPA@PBE, and vanishing within the error bars for EXX+RPA@PBE. One would expect that the cubic phase also prefers a ferroelectric order. Indeed, this is confirmed, with the $\mathrm{I}_{c}(\mathrm{a})$ being practically isoenergetic with the hexagonal ferroelectric phase. Along the sequence $\mathrm{I}_{c}(\mathrm{a}) \rightarrow \mathrm{I}_{c}(\mathrm{~d})$ the number of anti-ferroelectrically ordered neighbors increases. The energy increase is about equal for PBE and the two RPA variants, with a somewhat steeper increase in the RPA until $\mathrm{I}_{c}(\mathrm{c})$, and almost isoenergetic results for $\mathrm{I}_{c}(\mathrm{c})$ and $\mathrm{I}_{c}(\mathrm{~d})$ in the RPA. The important observation is that for the results at ambient pressure, PBE seems to capture all essential trends, and the RPA yields qualitatively and even quantitatively the same results. This is most likely related to the fact that the energy ordering is determined by the long range dipole-dipole interactions between different water molecules.

It is worthwhile mentioning that simple electrostatic non-polarizable water models predict that the antiferroelectric phase is more stable than the ferroelectric phase, 93 whereas DFT and more accurate methods predict that the ferroelectric order is preferred, both for the hexagonal as well as for the cubic phase. This can be related to the polarizability and additionally induced dipoles on the water molecules, which are neglected in simple rigid electrostatic water models.

The precision of PBE deteriorates quite dramatically, as one moves to high pressure phases. Pressure induces a sizable increase in the density and a reduction of the equilibrium volume. The energy ordering is shown in the top panel of Fig. 5. Let us start with a comparison between the present PBE calculations and previously published data. Generally our data reproduce the previous trends quite well, even though our data points tend to lie at slightly higher energies than the previous calculations. For ice VIII, the deviation amounts to about $20 \mathrm{meV}$. The reason for this discrepancy is that the present calculations were performed with a fairly large core oxygen PAW potential to reduce the computational cost for the subsequent RPA calculations. In any case, it is clear that compared to experiment both PBE calculations show a much too steep increase of the energy as the volumes decrease.

The RPA energy differences with respect to ice $\mathrm{I}_{h}$ agree very well with the previously published DMC data for ice II and ice VIII. Specifically, ice II becomes stabilized by almost $60 \mathrm{meV}$ compared to PBE, and the stabilization is even more dramatic for ice VIII where the energy difference decreases by almost $170 \mathrm{meV}$. There is a slight difference between the EXX+RPA@PBE and HF+RPA@PBE as the pressure increases: as already argued before, HF leads to a contraction of the density and therefore reduces the Pauli repulsion. Hence, the increase in the energy is smaller with HF+RPA@PBE than with EXX+RPA@PBE.

In our present calculations ice IX and ice II are almost iso-energetic lying about $10 \mathrm{meV}$ above the hexagonal disordered ice phase. We believe that this result is very reasonable and consistent with the experimental situation that both phases can be prepared at similar preparation conditions and pressures from different parent ice phases. The experimental estimates see ice II at slightly lower energies, but we can not confirm this (neither do the simulations using vdW corrected functionals). In the DMC the ice II is at lower energies than the disordered hexagonal phase, which is not confirmed by our RPA 


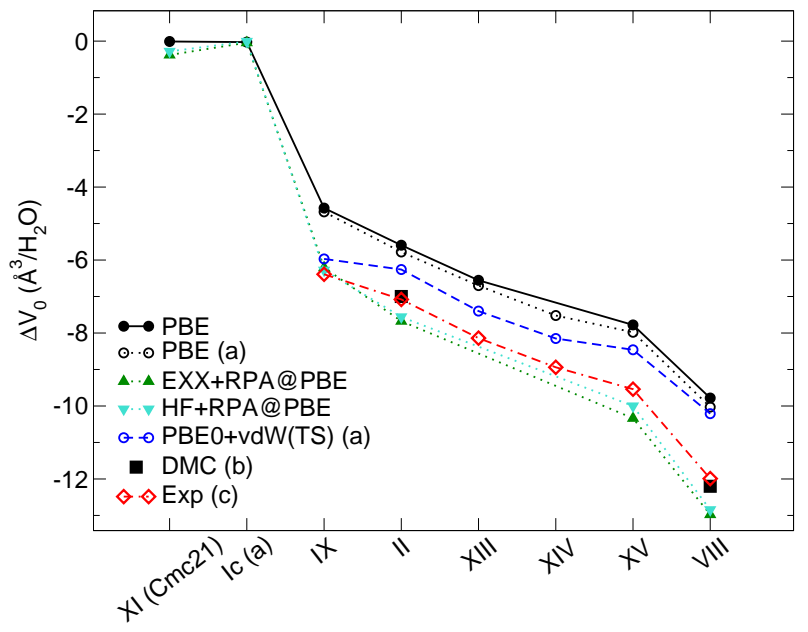

FIG. 6. Equilibrium volumes relative to ice $\mathrm{I}_{h} . \Delta V_{0}$ is the volume with respect to the $\mathrm{I}_{h}$ volume for the same method: $\Delta V_{0}=V_{0}$ (phase) $-V_{0}\left(\mathrm{I}_{h}\right)$. Other theoretical data marked with (a) and (b) are from Ref. 74 [PBE, PBE0-vdW(TS), DMC]. Experimental data (c) are compiled in Table III

data, either. However, considering the statistical error bars of the DMC calculations, we believe that this residual difference is not really meaningful.

For ice XV, our predicted energy differences agree very well with vdW corrected DFT calculations. However, for ice VIII, the increase in the energy is too steep using the vdW(TS) corrections. Here, the RPA does clearly better and yields energy changes in very good agreement with DMC simulations and experiment.

With respect to isolated water molecules, the RPA energies are not on par with the DMC results. From Table III we find for ice $\mathrm{I}_{h}$ a binding energy of $-545 \mathrm{meV}$ and $-680 \mathrm{meV}$ for EXX+RPA@PBE and HF+RPA@PBE, respectively. The experimental and DMC values are -610 and $-605 \pm 5 \mathrm{meV}$, respectively. Clearly the EXX+RPA underbinds by about $65 \mathrm{meV}$, whereas the HF+RPA overbinds by about the same amount. The correct value lies almost exactly in between the two values, suggesting that, in this case, a mean field description with $50 \%$ Hartree-Fock exchange and $50 \%$ EXX evaluated using DFT orbitals would yield very good results. This is a sensible result: in $\mathrm{H}_{2} \mathrm{O}$ and ice, the screening is very weak, which implies that hybrid functionals should include more exchange than in the typical hybrid functionals such as $\mathrm{PBE} 0 \underline{94}$ and HSE $\stackrel{95,96}{9}$ The qualitatively "best" orbitals are most likely obtained by a half-half hybrid functional, with "best" implying that such a functional yields a one-particle density matrix very close to the true one-particle density matrix.

\section{Equilibrium volumes}

The equilibrium volumes are summarized in Tab. III and Fig. 6. As expected from the energies, Tab. III sug- gests that $\mathrm{EXX}+\mathrm{RPA} @ \mathrm{PBE}$ again underbinds (too large volumes), whereas HF+RPA@PBE overbinds (too small volumes). As before, a mean field description with $50 \%$ Hartree-Fock exchange and $50 \%$ EXX employing DFT orbitals would yield very good volumes, confirming the final conjecture of the previous section. To delineate this constant volume error, we show in Fig. 6 the relative change of the volume with respect to disordered hexagonal ice $\mathrm{I}_{h}$. The problem of PBE is that the volume changes too little for the high pressure phase; from hexagonal or cubic ice to ice VIII only by $10 \AA^{3}$ per water molecule, whereas the experimental value is closer to $12 \AA^{3}$. Van der Waals corrections improve upon this value, but only slightly. In fact, they reduce all volumes by roughly the same fraction, so that changes in the relative volumes $\Delta V_{0}$ differ insignificantly between PBE and PBE-D.

The only method that yields almost exact results is DMC, but at a very steep compute cost. RPA exhibits a very good performance with little differences in the volume variations between EXX+RPA@PBE and HF+RPA@PBE. On first sight, the "compressibility" seems to be somewhat overestimated, with the volume changes being about $1 \AA^{3}$ too large for ice VIII. However, our data have not been corrected for zero point energy effects. Accounting for them increases the volumes and more so for the high pressure phases. The zero point expansion is about $5 \%$ stronger for ice VIII compared to ice $\mathrm{I}_{h}, \frac{8,74}{2}$ corresponding to roughly $1 \AA^{3}$. Including the zero point vibration effects will hence give almost perfect agreement in the volume changes for ice VIII with experiment.

\section{SUMMARY AND CONCLUSIONS}

The present work indicates that the random phase approximation yields a satisfactory description of ice for both the densities as well as the relative energies (compare Fig. 51). In principle, such a good description is not astonishing, since the random phase approximation seems to capture both van der Waals interactions as well as covalent bonding contributions reasonably well. Actually, when only the second order contribution is taken into account, the RPA reduces to the direct term in second order Møeller Plesset (MP2) perturbation theory, and MP2 is known to work very well for energy differences in water clusters.

From our point of view, RPA also improves upon simple pair-wise interaction potentials or vdW density functional theory. These two methods decrease the equilibrium volumes of ice compared to standard semi-local functionals by roughly the same amount for low and high density phases $\underline{\underline{74}}$ As a result, the volume change from hexagonal ice to ice VIII is too small. The RPA almost entirely mends this error (compare Fig. 6), in particular, when zero point vibration corrections are accounted for.

Up to date, only diffusion Monte Carlo was able to 
TABLE III. Equilibrium volumes in $\AA^{3} /$ molecule of the considered phases for PBE, EXX+RPA@PBE, HF+RPA@PBE, and experiment.

\begin{tabular}{lcrrrrr}
\hline \hline Phase & PBE & PBE0+vdW & EXX+RPA@PBE & HF+RPA@PBE & DMC & EXP \\
\hline I $_{h}$ & 30.23 & 29.88 & 32.81 & 31.31 & 31.69 & $32.05^{a}, 32.50^{b}$ \\
XI Cmc2 ${ }_{1}$ & 30.33 & & 32.43 & 31.03 & & $32.15^{c}, 31.92^{d}, 31.99^{e}$ \\
XI Pna2 & 30.23 & & 32.78 & 31.38 & & \\
I $_{c}$ (a) & 30.20 & & 32.77 & 31.53 & & $32.105^{b}$ \\
I $_{c}$ (b) & 30.20 & & 32.67 & 31.20 & & \\
I $_{c}(\mathrm{c})$ & 30.21 & & 32.64 & 30.87 & & \\
I $_{c}$ (d) & 30.23 & & 32.57 & 31.30 & & $25.80^{f}, 25.63^{g}$ \\
IX & 26.75 & & 27.32 & 25.60 & & $25.80^{f}, 25.63^{g}$ \\
IX c/a exp & 25.66 & 23.85 & 26.61 & 24.98 & & $24.97^{h}, 24.63^{i}$ \\
II & 24.63 & 23.63 & 25.14 & 23.76 & 24.7 & $23.91^{j}$ \\
XIII & 23.67 & 22.47 & & & & $22.53^{k}$ \\
XV & 22.45 & 21.45 & 22.48 & 21.32 & & $18.61^{l}, 20.09^{m}$ \\
VIII & 20.45 & 19.70 & 19.84 & 18.47 & 19.46 & \\
\hline \hline
\end{tabular}

${ }^{\mathrm{a}}$ Ref. 97

${ }^{\mathrm{b}}$ Ref. 98

${ }^{\mathrm{c}}$ Ref. 71

${ }^{\mathrm{d}}$ Ref. 72

${ }^{\text {e }}$ Ref. 99

${ }^{\mathrm{f}}$ Ref. 90

${ }^{\mathrm{g}}$ Ref. 91

${ }^{\mathrm{h}}$ Ref. 100

${ }^{i}$ Ref. 101

${ }^{j}$ Ref. 84

${ }^{\mathrm{k}}$ Ref. 85

${ }^{1}$ Ref. 88

${ }^{\mathrm{m}}$ Ref. 89

attain a similar - or more precisely — slightly superior description, but as already emphasized, at a very steep computational cost. RPA achieves results that are close to DMC, but at a fraction of the computational cost. For instance, the total compute time for the rather complicated ice XIII phase (28 molecules) is about 4 hours on 64 cores, and the compute time for ice IX (12 molecules) about 2 hours on 16 cores (both calculations performed using $2 \times 2 \times 2 \mathrm{k}$-points). These favorable timings were achieved with a not yet released RPA code that scales cubically with system size and linearly with the number of k-points. Given the favorable results for the energetics obtained here and these favourable timings, we believe an $a b$ initio treatment of ice on surfaces is now within reach. The only downside of the random phase approximation is that the ice binding energies with respect to isolated water molecules are in error by about $50 \mathrm{meV}$.

In the present work, we have used two RPA flavors: the usual combination of EXX+RPA@PBE, where both the RPA correlation and the EXX are evaluated using the same DFT orbitals, and the combination of RPA@PBE with exact Hartree-Fock energies. The first method underestimates the binding energies and overestimates all predicted volumes. The second approximation overestimates the binding energy and underestimates the equilibrium volumes. A simple solution to this problem is to determine the exact exchange energy from orbitals obtained with a so called half-half functional, a functional where half the HF exchange and half the density functional theory exchange is used. The final results are merely in-between the two limiting approximations considered here and in almost perfect agreement with the experimental and DMC results.

Acknowledgment: This work was supported by the Austrian Science Fund (FWF) within the SFB ViCoM (Grant F 41). We thank Biswajit Santra for providing us with the ice structures. C.F. and M.M. thank Gianni Profeta for providing useful structural information on ice XI. Supercomputing time on the Vienna Scientific cluster (VSC) is gratefully acknowledged.
1 C. Lee, D. Vanderbilt, K. Laasonen, R. Car, and M. Parrinello, Phys. Rev. Lett. 69, 462 (1992)
2 S. J. Singer, J.-L. Kuo, T. K. Hirsch, C. Knight, L. Ojamäe, and M. L. Klein, Phys. Rev. Lett. 94, 135701 
(2005)

3 G. A. Tribello, B. Slater, and C. G. Salzmann, J. Am. Chem. Soc. 128, 12594 (2006)

${ }^{4}$ P. J. Feibelman, Phys. Chem. Chem. Phys. 10, 4688 (2008)

5 A. Hermann and P. Schwerdtfeger, Phys. Rev. Lett. 101, 183005 (2008)

6 I. Hamada, J. Chem. Phys. 133, 214503 (2010)

7 B. Santra, J. Klimeš, D. Alfè, A. Tkatchenko, B. Slater, A. Michaelides, R. Car, and M. Scheffler, Phys. Rev. Lett. 107, 185701 (2011)

8 E. D. Murray and G. Galli, Phys. Rev. Lett. 108, 105502 (2012)

9 B. Pamuk, J. M. Soler, R. Ramírez, C. P. Herrero, P. Stephens, P. B. Allen, and M.-V. Fernández-Serra, Phys. Rev. Lett. 108, 193003 (2012)

10 K. Laasonen, M. Sprik, M. Parrinello, and R. Car, J. Chem. Phys. 99, 9080 (1993)

11 M. Sprik, J. Hutter, and M. Parrinello, J. Chem. Phys. 105, 1142 (1996)

12 P. L. Silvestrelli and M. Parrinello, J. Chem. Phys. 111, 3572 (1999)

13 B. Chen, I. Ivanov, M. L. Klein, and M. Parrinello, Phys. Rev. Lett. 91, 215503 (2003)

14 D. Asthagiri, L. R. Pratt, and J. D. Kress, Phys. Rev. E 68, 041505 (2003)

15 J. C. Grossman, E. Schwegler, E. W. Draeger, F. Gygi, and G. Galli, J. Chem. Phys. 120, 300 (2004)

16 E. Schwegler, J. C. Grossman, F. Gygi, and G. Galli, J. Chem. Phys. 121, 5400 (2004)

17 M. V. Fernández-Serra and E. Artacho, J. Chem. Phys. 121, 11136 (2004)

18 I.-F. W. Kuo, C. J. Mundy, M. J. McGrath, J. I. Siepmann, J. VandeVondele, M. Sprik, J. Hutter, B. Chen, M. L. Klein, F. Mohamed, M. Krack, and M. Parrinello, J. Phys. Chem. B 108, 12990 (2004)

19 M. J. McGrath, J. I. Siepmann, I.-F. W. Kuo, C. J. Mundy, J. VandeVondele, J. Hutter, F. Mohamed, and M. Krack, J. Phys. Chem. A 110, 640 (2006)

${ }^{20}$ T. Todorova, A. P. Seitsonen, J. Hutter, I.-F. W. Kuo, and C. J. Mundy, J. Phys. Chem. B 110, 3685 (2006)

21 H.-S. Lee and M. E. Tuckerman, J. Chem. Phys. 125, 154507 (2006)

22 J. A. Morrone and R. Car, Phys. Rev. Lett. 101, 017801 (2008)

23 T. D. Kühne, M. Krack, and M. Parrinello, J. Chem. Theory Comput. 5, 235 (2009)

24 S. Yoo, X. C. Zeng, and S. S. Xantheas, J. Chem. Phys. 130, 221102 (2009)

25 I.-C. Lin, A. P. Seitsonen, M. D. Coutinho-Neto, I. Tavernelli, and U. Rothlisberger, J. Phys. Chem. B 113, 1127 (2009)

26 J. Schmidt, J. VandeVondele, I.-F. W. Kuo, D. Sebastiani, J. I. Siepmann, J. Hutter, and C. J. Mundy, J. Phys. Chem. B 113, 11959 (2009)

27 C. Zhang, D. Donadio, F. Gygi, and G. Galli, J. Chem. Theory Comput. 7, 1443 (2011)

${ }^{28}$ O. Akin-Ojo and F. Wang, Chem. Phys. Lett. 513, 59 (2011)

29 J. Wang, G. Román-Pérez, J. M. Soler, E. Artacho, and M.-V. Fernández-Serra, J. Chem. Phys. 134, 024516 (2011)

30 S. Yoo and S. S. Xantheas, J. Chem. Phys. 134, 121105 (2012)
31 C. Zhang, J. Wu, G. Galli, and F. Gygi, J. Chem. Theory Comput. 7, 3054 (2011)

${ }^{32}$ K. Kim and K. D. Jordan, J. Phys. Chem. 98, 10089 (1994)

33 S. S. Xantheas, J. Chem. Phys. 102, 4505 (1995)

${ }^{34}$ S. S. Xantheas, C. J. Burnham, and R. J. Harrison, J. Chem. Phys. 116, 116 (2002)

35 B. Santra, A. Michaelides, and M. Scheffler, J. Chem. Phys. 127, 184104 (2007)

36 B. Santra, A. Michaelides, and M. Scheffler, J. Chem. Phys. 131, 124509 (2009)

37 E. E. Dahlke, R. M. Olson, H. R. Leverentz, and D. G. Truhlar, J. Phys. Chem. A 112, 3976 (2008)

38 G. C. Shields and K. N. Kirschner, Synthesis and Reactivity in Inorganic, Metal-Organic, and Nano-Metal Chemistry $\mathbf{3 8}, 32$ (2008)

39 J. R. Hammond, N. Govind, K. Kowalski, J. Autschbach, and S. S. Xantheas, J. Chem. Phys. 131, 214103 (2009)

40 F.-F. Wang, G. Jenness, W. A. Al-Saidi, and K. D. Jordan, J. Chem. Phys. 132, 134303 (2010)

41 R. M. Shields, B. Temelso, K. A. Archer, T. E. Morrell, and G. C. Shields, J. Phys. Chem. A 114, 11725 (2010)

42 P. J. Bygrave, N. L. Allan, and F. R. Manby, J. Chem. Phys. 137, 164102 (2012)

${ }^{43}$ M. J. Gillan, F. R. Manby, M. D. Towler, and D. Alfè, J. Chem. Phys. 136, 244105 (2012)

${ }^{44}$ M. J. Gillan, D. Alfè, P. J. Bygrave, C. R. Taylor, and F. R. Manby, J. Chem. Phys. 139, 114101 (2013)

45 M. D. Ben, M. Schönherr, J. Hutter, and J. VandeVondele, J. Phys. Chem. Lett. 4, 3753 (2013)

${ }^{46}$ M. D. Ben, J. Hutter, and J. VandeVondele, J. Chem. Theo. Comput. 9, 2654 (2013)

47 D. P. O'Neill, N. L. Allan, and F. R. Manby, in Accurate Condensed-Phase Quantum Chemistry, edited by F. Manby (CRC Press, Taylor \& Francis Group, Boca Raton, 2010)

48 M. Dion, H. Rydberg, E. Schröder, D. C. Langreth, and B. I. Lundqvist, Phys. Rev. Lett. 92, 246401 (2004)

49 D. C. Langreth, M. Dion, H. Rydberg, E. Schroder, P. Hyldgaard, and B. I. Lundqvist, Int. J. Quant. Chem. 101, 599 (2005)

50 A. K. Kelkkanen, B. I. Lundqvist, and J. K. Nørskov, J. Chem. Phys. 131, 046102 (2009)

51 S. Grimme, J. Comput. Chem. 27, 1787 (2006)

52 A. Tkatchenko and M. Scheffler, Phys. Rev. Lett. 102, 073005 (2009)

53 B. Santra, A. Michaelides, M. Fuchs, A. Tkatchenko, C. Filippi, and M. Scheffler, J. Chem. Phys. 129, 194111 (2008)

54 J. Klimeš and A. Michaelides, J. Chem. Phys. 137, 120901 (2012)

55 J. Carrasco, B. Santra, J. Klimeš, and A. Michaelides, Phys. Rev. Lett. 106, 026101 (2011)

56 J. Carrasco, A. Hodgson, and A. Michaelides, Nature Mater. 11, 667 (2012)

57 J. Carrasco, J. Klimeš, and A. Michaelides, J. Chem. Phys. 138, 024708 (2013)

58 J. Harl and G. Kresse, Phys. Rev. B 77, 045136 (2008)

59 S. Lebègue, J. Harl, T. Gould, J. G. Ángyán, G. Kresse, and J. F. Dobson, Phys. Rev. Lett. 105, 196401 (2010)

${ }^{60}$ X. Ren, A. Tkatchenko, P. Rinke, and M. Scheffler, Phys. Rev. Lett. 106, 153003 (2011)

61 J. Harl and G. Kresse, Phys. Rev. Lett. 103, 056401 
(2010)

62 J. Harl, L. Schimka, and G. Kresse, Phys. Rev. B 81, $115126(2010)$

63 L. Schimka, R. Gaudoin, J. Klimeš, M. Marsman, and G. Kresse, Phys. Rev. B 87, 214102 (2013)

64 J. Yan, J. S. Hummelshøj, and J. K. Nørskov, Phys. Rev. B 87, 075207 (2013)

65 P. E. Blöchl, Phys. Rev. B 50, 17953 (1994)

66 G. Kresse and J. Joubert, Phys. Rev. B 59, 1758 (1999)

67 J. P. Perdew, K. Burke, and M. Ernzerhof, Phys. Rev. Lett. 77, 3865 (1996), ibid, 78, 1396 (1997)

68 J. Paier, X. Ren, P. Rinke, G. E. Scuseria, A. Grüneis, G. Kresse, and M. Scheffler, New J. Phys. 14, 043002 (2012)

69 X. Ren, P. Rinke, V. Blum, J. Wieferink, A. Tkatchenko, A. Sanfilippo, K. Reuter, and M. Scheffler, New J. Phys. 14, 053020 (2012)

70 X. Ren, P. Rinke, G. E. Scuseria, and M. Scheffler, Phys. Rev. B 88, 035120 (2013)

71 A. J. Leadbetter, R. C. Ward, J. W. Clark, P. A. Tucker, T. Matsuo, and H. Suga, J. Chem. Phys. 82, 424 (1985)

72 R. Howe and R. W. Whitworth, J. Chem. Phys. 90, 4450 (1989)

73 D. R. Hamann, Phys. Rev. B 55, R10157 (1997)

74 B. Santra, J. Klimeš, A. Tkatchenko, D. Alfè, B. Slater, A. Michaelides, R. Car, and M. Scheffler, J. Chem. Phys. 139, 154702 (2013)

75 E. R. Davidson and K. Morokuma, J. Chem. Phys. 81, 3741 (1984)

76 T. K. Hirsch and L. Ojamäe, J. Phys. Chem. B 108, 15856 (2004)

77 G. Profeta and S. Scandolo, Phys. Rev. B 84, 024103 (2011)

78 B. J. Murray, D. A. Knopf, and A. K. Bertram, Nature 434, 202 (2005)

79 Z. Raza, D. Alfé, C. G. Salzmann, J. Klimeš, A. Michaelides, and B. Slater, Phys. Chem. Chem. Phys. 13, 19788 (2011)

80 P. Geiger, C. Dellago, M. Macher, C. Franchini, G. Kresse, J. Bernard, J. N. Stern, and T. Loerting submitted

81 E. Whalley, J. B. R. Heath, and D. W. Davidson,
J. Chem. Phys. 48, 2362 (1968)

82 B. Kamb, Acta Crystallographica 17, 1437 (1964)

83 M. Chaplin, "Water phase diagram," (2012), http://www.lsbu.ac.uk/water/phase.html, accessed 2. 7. 2012

84 C. G. Salzmann, Science 311, 1758 (2006)

85 C. Salzmann, P. Radaelli, E. Mayer, and J. Finney, Phys. Rev. Lett. 103, 105701 (2009)

${ }^{86}$ W. F. Kuhs, J. L. Finney, C. Vettier, and D. V. Bliss, J. Chem. Phys. 81, 3612 (1984)

87 P. Pruzan, J. C. Chervin, and B. Canny, J. Chem. Phys. 99, $9842(1993)$

88 J. D. Jorgensen, R. A. Beyerlein, N. Watanabe, and T. G. Worlton, J. Chem. Phys. 81, 3211 (1984)

${ }^{89}$ Y. Yoshimura, S. T. Stewart, M. Somayazulu, H.-k. Mao, and R. J. Hemley, J. Chem. Phys. 124, 024502 (2006)

90 S. J. La Placa, W. C. Hamilton, B. Kamb, and A. Prakash, J. Chem. Phys. 58, 567 (1973)

91 J. D. Londono, W. F. Kuhs, and J. L. Finney, J. Chem. Phys. 98, 4878 (1993)

92 E. Whalley, J. Chem. Phys. 81, 4087 (1984)

93 J. Lekner, Physica B: Condensed Matter 240, 263 (1997)

94 C. Adamo and V. Barone, J. Chem. Phys. 110, 6158 (1999)

95 J. Heyd, G. E. Scuseria, and M. Ernzerhof, J. Chem. Phys. 118, 8207 (2003)

96 J. Heyd, G. E. Scuseria, and M. Ernzerhof, J. Chem. Phys. 124, 219906 (2006)

97 P. V. Hobbs, Ice physics, Oxford classic texts in the physical sciences (Oxford University Press, New York, 1974) ISBN 9780199587711

98 C. Vega, C. McBride, E. Sanz, and J. L. F. Abascal, Phys. Chem. Chem. Phys. 7, 1450 (2005)

99 C. M. B. Line and R. W. Whitworth, J. Chem. Phys. 104, 10008 (1996)

100 A. D. Fortes, I. G. Wood, M. Alfredsson, L. Vočadlo, and K. S. Knight, Journal of Applied Crystallography 38, 612 (2005)

101 C. Lobban, J. L. Finney, and W. F. Kuhs, J. Chem. Phys. 117, $3928(2002)$ 